\title{
Numerical Convolution Method in Time Domain and Its Application to Nonrigid Earth Nutation Theory
}

\author{
Toshio Fukushima \\ National Astronomical Observatory, 2-21-1, Ohsawa, Mitaka, Tokyo \\ 181-8588, Japan; Toshio.Fukushima@nao.ac.jp \\ Toshimichi Shirai \\ University of Tokyo, School of Science, Department of Astronomy, \\ 3-8-1, Hongoh, Bunkyo-ku, Tokyo 113-0033, Japan
}

\begin{abstract}
We developed a numerical method to incorporate nonrigid effects into a nutation theory of the rigid Earth. Here we assume that the nonrigid effects are based on a linear response theory and its transfer function is expressed as a rational function of frequency. The method replaces the convolution of the transfer function in the frequency domain by the corresponding integro-differential operations in the time domain numerically; namely multiplying the polynomial in the frequency domain by the numerical differentiations in the time domain and multiplying the fractions in the frequency domain by the numerical integrations with a suitable kernel in the time domain. In replacing by the integrations, the method requires the determination of the coefficients of free oscillation. This is done by a least-squares method to fit the theory incorporated with the nonrigid effects to the observational data, whose availability is also assumed. The numerical differentiation and integration are effectively computed by means of the symmetric formulas of differentiation and integration. Numerical tests showed that the method is sufficiently precise to reproduce the analytically convolved nutation at the level of 10 nano arcseconds by using the 9-point central difference formulas and the 8point symmetric integration formula to cover the period of 15 years with 1.5-hour stepsize. Since we only require the rigid Earth nutation theory to be expressed as a numerical table of time, this method enables one to create a purely numerical theory of nutation of the nonrigid Earth.
\end{abstract}

\section{Introduction}

Few of the nutation theories of the nonrigid Earth are fully numerical. For example, the current IAU Theory of Nutation (Seidelmann 1982) is the combination of an analytical theory of nutation of the rigid Earth (Kinoshita 1977) and an analytical treatment of nonrigid effects based on a linear response theory of the Earth (Wahr 1981). The nonrigid effects are introduced by the analytical convolution of the transfer function to the nutation theory of the rigid Earth. Here the convolution is done in the frequency domain since the transfer function 
is expressed as a rational function of frequency while the rigid Earth nutation theory is given as Fourier series with respect to time.

This approach has been followed by almost all of the researchers. See the report of the IAU/IUGG Working Group on Nutation (Dehant et al. 1999). Some created new analytical theories of the rigid Earth nutation (Kinoshita and Souchay 1990; Bretagnon et al. 1997; Roosbeek and Dehant, 1998). Others developed new transfer functions of the nonrigid Earth (Mathews et al., 1991a; Mathews et al., 1991b; Herring, 1995; Schastok, 1997; Mathews et al., 1999). Anyway, unchanged has been the way of combining these two factors; the analytical convolution. Recently, Getino et al. (1999) developed a scheme to obtain the nutation of the nonrigid Earth directly by solving the rotational equations of the multi-component Earth. However, it is also analytical.

On the other hand, most of the state-of-the-art theories of orbital motions of the planets and their major satellites are fully numerical. The state-of-the-art theory of the rotational motion of the Moon is also numerical. Good examples are JPL's DE series. This is simply because the numerical theories are more precise in the sense of explaining observational data better.

Then, there comes a naive question. Why do not we have a fully numerical theory of nutation? The major reason is that the above analytical approach has been the only method known to execute the convolution precisely and efficiently. Of course, the discrete Fourier transform of a numerically integrated nutation of the rigid Earth can be done quickly by means of FFT. However, we learned that the end effects, which are caused by the fact that the integrated nutation is not single-periodic, introduce spurious components into the Fourier spectrum, and therefore degrade the precision of the Fourier transform significantly. On the other hand, if we allow the transform to be multi-periodic, then the problem finally reduces to a large-scale nonlinear optimization, which would require a tremendous amount of computational time.

In this short article, we report that a purely numerical method of convolution works if (1) the transfer function expressed in the frequency domain is of rational form and (2) the observational data of nutation to be fitted are availlable. Since the method requires the rigid Earth nutation theory only to be expressed as a numerical table of time, it enables us to create a fully numerical nutation theory of the nonrigid Earth, which would be consistent with the latest lunar and planetary ephemerides such as JPL's DE series.

\section{Method}

\subsection{Outline}

Consider convolving a function expressed in the frequency domain $g(\omega)$ and a function expressed in the time domain $z(t)$ to obtain another function in the time domain $\zeta(t)$.

In the language of the nutation theory, the function $g(\omega)$ is the transfer function of the nonrigid Earth. Its example is of the form of a rational function as

$$
g(\omega)=\sum_{k=0}^{K} A_{k} \omega^{k}+\sum_{j=1}^{J} \frac{B_{j}}{\omega-\omega_{j}}
$$


Table 1. Some Parameters of Transfer Functions

\begin{tabular}{lcccccc}
\hline \hline Model & $A_{0}$ & $A_{1}$ & $B_{1}$ & $\omega_{1}-\Omega$ & $B_{2}$ & $\omega_{2}$ \\
\hline Wahr & +1.0497 & -0.282 & -6.038 & +2.480 & -1.091 & -2.174 \\
Dehant \& Defraigne & +1.0504 & -0.279 & -6.070 & +2.485 & -1.190 & -2.316 \\
Herring (Re) & +1.0490 & -0.255 & -5.799 & +2.532 & -1.149 & -2.319 \\
Herring (Im) & -0.0015 & +0.040 & - & - & -0.021 & +0.029 \\
\hline
\end{tabular}

Note: Shown are the rounded values of some parameters of the existing transfer functions. Those of Mathews et al. (1999) are not shown since they contain some free parameters. The units of the coeffcients are 1 for $A_{0}, \Omega$ for $A_{1}, 10^{-4} \Omega$ for $B_{j}(j=1,2)$, and $10^{-3} \Omega$ for $\omega_{1}-\Omega$ and $\omega_{2}$, respectively. Here $\Omega=7.292115 \times 10^{-5} \mathrm{rad} / \mathrm{s}$ is the nominal mean angular velocity of the Earth.

where the coefficients, $A_{k}$ and $B_{j}$, are complex numbers in general, $\omega_{j}$ are the complex eigenfrequencies ${ }^{1}$ of the nonrigid Earth regarded as a linearly responding body, and $i \equiv \sqrt{-1}$. Typical examples of the transfer functions are;

1. that of Wahr (1981) where $K=J=2$ while $A_{k}, B_{j}$, and $\omega_{j}$ are all real,

2. that of Dehant and Defraigne ${ }^{2}$ (1997) where $K=J=2$ while $A_{k}, B_{j}$, and $\omega_{j}$ are all real,

3. that of Herring (1995) where $K=1$ and $J=2$ while $A_{k}, B_{1}$, and $\omega_{1}$ are complex and $B_{2}$ and $\omega_{2}$ are real, and

4. that of Mathews et al. (1999) where $K=1$ and $J=4$ while $A_{k}, B_{j}$, and $\lambda_{j}$ are all complex.

See Table 1 for the numerical values of these major parameters.

On the other hand, the functions $z(t)$ and $\zeta(t)$ are the complex forms of the nutation of the rigid and the nonrigid Earth, respectively; namely

$$
z(t)=\Delta \epsilon_{\mathrm{R}}-i s_{0} \Delta \psi_{\mathrm{R}}, \quad \zeta(t)=\Delta \epsilon_{\mathrm{NR}}-i s_{0} \Delta \psi_{\mathrm{NR}}, \quad s_{0} \equiv \sin \epsilon_{0},
$$

where $\Delta \psi$ and $\Delta \epsilon$ are the nutations in longitude and in obliquity, respectively, the subscripts $\mathrm{R}$ and NR denote the rigid and nonrigid Earth, respectively, and $\epsilon_{0}$ is the mean obliquity of ecliptic at the epoch J2000.0 (Kinoshita 1977). In place of $s_{0}$, which is a constant of time, some authors used $s \equiv \sin \epsilon$, where $\epsilon$ is the mean obliquity of ecliptic, which is usually expressed as a low order polynomial of time (Seidelmann 1982).

Now, if the function $z(t)$ is expressed as Fourier series

$$
z(t)=\sum_{\ell} z_{\ell} e^{i \Omega_{\ell} t}
$$

then the convolution is straightforward and the convolved function is obtained as

$$
\zeta(t)=\sum_{\ell} \zeta_{\ell} e^{i \Omega_{\ell} t}
$$

\footnotetext{
${ }^{1}$ By tradition, the eigenfrequencies close to 1 are called wobbles like Chandler Wobble (CW), while those close to 0 are called nutations like Free Core Nutation (FCN).

${ }^{2}$ They did not give the expression of $A_{2}$ in this form although it is easily derived from their original expression.
} 
Table 2. Various Effects in Convolution of Rigid-Earth Nutations

\begin{tabular}{llr}
\hline \hline Effect & Details & Magnitude \\
\hline Mixed Secular Terms & - & 3 mas \\
Round-off & (published) vs. (computed) & 0.5 mas \\
Delauney Arguments & Model Difference (Newcomb vs. VSOP) & $40 \mu \mathrm{as}$ \\
& High-Order Trends & $30 \mu \mathrm{as}$ \\
Numerical & Integration Error (FCN) & $0.1 \mu \mathrm{as}$ \\
& Differentiation Error & 8 nas \\
& Integration Error (CW) & 5 nas \\
\hline
\end{tabular}

Note: Shown are the order of magnitudes of various effects to be considered in conducting the convolution of rigid-Earth nutation. The effects are evaluated numerically for the period of 1984-1999 and their maximum of the peak-to-peak values are listed. Remark that the numerical errors can be reduced by choosing a smaller stepsize or by adopting a higher order formula.

where

$$
\zeta_{\ell}=g\left(\Omega_{\ell}\right) z_{\ell}
$$

This is the analytical way of convolution.

We remark that the analytical convolutions conducted so far are not rigorous. In fact, the treatment of the mixed secular terms like $t e^{i \omega t}$ appearing in many analytical theories of the rigid Earth nutation has been incomplete. Also questionable is the effect of higher-order $\left(t^{2}, t^{3}\right.$, and $\left.t^{4}\right)$ secular terms of the Delauney arguments. See Table 2 for the order of magnitudes of these effects.

Now, what we aim for is to do the convolution without assuming the Fourier expressions of $z(t)$. More specifically, we seek a numerical method to do this convolution under only the condition that $z(t)$ is given as a table of its values at equally-spaced times. Since the convolution is a linear operation, we only have to combine the results of convolutions of each element. Then we will discuss each element below.

\subsection{Powers}

Consider the case that the transfer function is a power of $\omega$,

$$
g(\omega)=\omega^{k} .
$$

This form in the frequency domain is easily translated to the differential operation in the time domain as

$$
\omega^{k} \rightarrow\left(-i \frac{\mathrm{d}}{\mathrm{dt}}\right)^{k}
$$

In fact,

$$
\frac{\mathrm{d} e^{i \Omega t}}{\mathrm{dt}}=i \Omega e^{i \Omega t}, \quad \frac{\mathrm{d}^{2} e^{i \Omega t}}{\mathrm{dt}^{2}}=-\Omega^{2} e^{i \Omega t}, \quad \ldots
$$

Assume that the values of $z(t)$ are tabulated at equally-spaced grid points of time as

$$
z_{n}=z\left(t_{n}\right), \quad t_{n}=t_{0}+n h,
$$


where $h$ is the stepsize of tabulation. Then the table of its first order derivatives, $z_{n}^{(1)}$, are numerically obtained by the first order central difference formulas;

$$
\begin{gathered}
2 h z_{n}^{(1)}=z_{n+1}-z_{n-1}+O\left(h^{3}\right) . \\
12 h z_{n}^{(1)}=8\left(z_{n+1}-z_{n-1}\right)-\left(z_{n+2}-z_{n-2}\right)+O\left(h^{5}\right) . \\
60 h z_{n}^{(1)}=45\left(z_{n+1}-z_{n-1}\right)-9\left(z_{n+2}-z_{n-2}\right)+\left(z_{n+3}-z_{n-3}\right)+O\left(h^{7}\right) . \\
840 h z_{n}^{(1)}=672\left(z_{n+1}-z_{n-1}\right)-168\left(z_{n+2}-z_{n-2}\right) \\
+32\left(z_{n+3}-z_{n-3}\right)-3\left(z_{n+4}-z_{n-4}\right)+O\left(h^{9}\right) .
\end{gathered}
$$

One must prepare the table of $z_{n}$ for a little longer period than the period of the table of $z_{n}^{(1)}$ required.

In obtaining higher order derivatives, one may use the above formulas repeatedly. However, there are other types of formulas which directly evaluate them. One such example is that for the second order one, $z_{n}^{(2)}$, as

$$
\begin{gathered}
h^{2} z_{n}^{(2)}=-2 z_{n}+\left(z_{n+1}+z_{n-1}\right)+O\left(h^{4}\right) . \\
12 h^{2} z_{n}^{(2)}=-18 z_{n}+8\left(z_{n+1}+z_{n-1}\right)+\left(z_{n+2}+z_{n-2}\right)+O\left(h^{6}\right) . \\
240 h^{2} z_{n}^{(2)}=-340 z_{n}+145\left(z_{n+1}+z_{n-1}\right)+26\left(z_{n+2}+z_{n-2}\right) \\
-\left(z_{n+3}+z_{n-3}\right)+O\left(h^{8}\right) .
\end{gathered}
$$

The above formulas include many differences of the quantities of the same order of magnitude. Thus one must take care of the loss of precision. If the tables $z_{n}$ are obtained from numerical integrations directly, it is better to save some intermediate variables such as the backward differences, $\nabla z_{n} \equiv z_{n}-z_{n-1}$, or the squared central differences, $\delta^{2} z_{n} \equiv z_{n+1}-2 z_{n}+z_{n-1}$, and to use them instead of the integrated $z_{n}$.

\subsection{First Order Fractions}

Consider the case of first order fractions,

$$
g(\omega)=\frac{1}{\omega+i \lambda} .
$$

This form is translated by means of the indefinite integral operation with a Green kernel as

$$
\frac{1}{\omega+i \lambda} \rightarrow i e^{\lambda t} \int^{t} \mathrm{~d} s e^{-\lambda s}
$$

In fact, it is straightforward to show that

$$
i e^{\lambda t} \int^{t} e^{-\lambda s} e^{i \omega s} \mathrm{~d} s=\frac{e^{i \omega t}}{\omega+i \lambda}+C e^{\lambda t}
$$


where $C$ is an integral constant. Apart from the determination of the integral constant, which will be discussed in the next subsection, we first discuss how to obtain the integral. Since the indefinite integral is not realized numerically, we consider evaluating the definite integral

$$
x_{n} \equiv \int_{t_{0}}^{t_{n}} v(s) \mathrm{d} s, \quad v(s) \equiv e^{-\lambda s} z(s),
$$

from the tables of

$$
v_{n} \equiv v\left(t_{n}\right)=e^{-\lambda t_{n}} z_{n} .
$$

This is realized by the numerical integration with the initial condition

$$
x_{0}=0 \text {. }
$$

There we use the symmetric integration formulas as

$$
\begin{gathered}
2\left(x_{n+1}-x_{n}\right)=h\left(v_{n+1}+v_{n}\right)+O\left(h^{3}\right) . \\
24\left(x_{n+1}-x_{n}\right)=h\left[13\left(v_{n+1}+v_{n}\right)-\left(v_{n+2}+v_{n-1}\right)\right]+O\left(h^{5}\right) . \\
1440\left(x_{n+1}-x_{n}\right)=h\left[802\left(v_{n+1}+v_{n}\right)-93\left(v_{n+2}+v_{n-1}\right)\right. \\
\left.+11\left(v_{n+3}+v_{n-2}\right)\right]+O\left(h^{7}\right) . \\
120960\left(x_{n+1}-x_{n}\right)=h\left[66413\left(v_{n+1}+v_{n}\right)-9631\left(v_{n+2}+v_{n-1}\right)\right. \\
\left.+1879\left(v_{n+3}+v_{n-2}\right)-191\left(v_{n+4}+v_{n-3}\right)\right]+O\left(h^{9}\right) .
\end{gathered}
$$

\subsection{Determination of Integral Constants}

Now, consider how to determine the integral constants. After collecting the formulas derived in the previous subsections, we know that the tables of $\zeta$ are expressed as

$$
\zeta_{n}=\sum_{k=0}^{K} A_{k}(-i)^{k} z_{n}^{(k)}+i \sum_{j=1}^{J} B_{j} \xi_{n}^{(j)}+\sum_{\ell=1}^{J} C_{\ell} \eta_{n}^{(\ell)}
$$

where

$$
\xi_{n}^{(j)}=\eta_{n}^{(j)} x_{n}^{(j)}, \quad \eta_{n}^{(j)}=e^{i \omega_{j} t_{n}}, \quad(j=1, \cdots, J)
$$

and

$$
x_{n}^{(j)}=\int_{t_{0}}^{t_{n}} v^{(j)}(s) \mathrm{d} s, \quad v^{(j)}(s)=e^{-i \omega_{j} s} z(s), \quad(j=1, \cdots, J) .
$$

Assume that (1) the coefficients $A_{k},(k=0, \cdots, K)$, and $B_{j},(j=1, \cdots, J)$, as well as the eigenfrequencies $\omega_{j},(j=1, \cdots, J)$, are all known and that (2) the observational data, $\hat{\zeta}_{n}$, to which the convolved functions are to be fitted, and their weights, $w_{n}$, are available at $t_{n}$. Then we can determine the unknown complex parameters $C_{j}$ by a simple least-squares method. 
Consider minimizing the chi square with complex parameters $C_{j}$

$$
\chi^{2} \equiv \sum_{n=0}^{N} w_{n}\left|\left(\sum_{j=1}^{J} C_{j} \eta_{n}^{(j)}\right)-\Delta \zeta_{n}\right|^{2}
$$

where

$$
\Delta \zeta_{n}=\hat{\zeta}_{n}-\left(\sum_{k=0}^{K} A_{k}(-i)^{k} z_{n}^{(k)}+i \sum_{j=1}^{J} B_{j} \xi_{n}^{(j)}\right) .
$$

Then the summand is expanded as

$$
\begin{gathered}
\left|\left(\sum_{j=1}^{J} C_{j} \eta_{n}^{(j)}\right)-\Delta \zeta_{n}\right|^{2} \\
=\left[\left(\sum_{j=1}^{J} C_{j} \eta_{n}^{(j)}\right)-\Delta \zeta_{n}\right]\left[\left(\sum_{k=1}^{J} C_{k}^{*}\left(\eta_{n}^{(k)}\right)^{*}\right)-\left(\Delta \zeta_{n}\right)^{*}\right] \\
=\left[\sum_{j=1}^{J} \sum_{k=1}^{J} C_{j} C_{k}^{*} \eta_{n}^{(j)}\left(\eta_{n}^{(k)}\right)^{*}\right]-\left[\sum_{j=1}^{J} C_{j} \eta_{n}^{(j)}\left(\Delta \zeta_{n}\right)^{*}\right] \\
-\left[\sum_{k=1}^{J} C_{k}^{*}\left(\eta_{n}^{(k)}\right)^{*} \Delta \zeta_{n}\right]+\left|\Delta \zeta_{n}\right|^{2},
\end{gathered}
$$

where $^{*}$ denotes a complex conjugate. The conditions of minimization are

$$
\left(\frac{\partial \chi^{2}}{\partial C_{j}}\right)_{\left(C_{k}\right)^{*}}=\left[\left(\frac{\partial \chi^{2}}{\partial\left(C_{j}\right)^{*}}\right)_{C_{k}}\right]^{*}=0 .
$$

These are no other than the normal equation,

$$
\sum_{k=1}^{J} d_{j k} C_{k}=e_{j}, \quad(j=1, \cdots, J)
$$

where

$$
d_{j k}=\sum_{n=0}^{N} w_{n}\left(\eta_{n}^{(j)}\right)^{*} \eta_{n}^{(k)}, \quad e_{j}=\sum_{n=0}^{N} w_{n}\left(\eta_{n}^{(j)}\right)^{*} \Delta \zeta_{n} .
$$

Thus, the coefficients $C_{j}$ are obtained by solving these $J \times J$ complex linear equation by the Gauss-Jordan or other methods to solve a set of linear equations. 


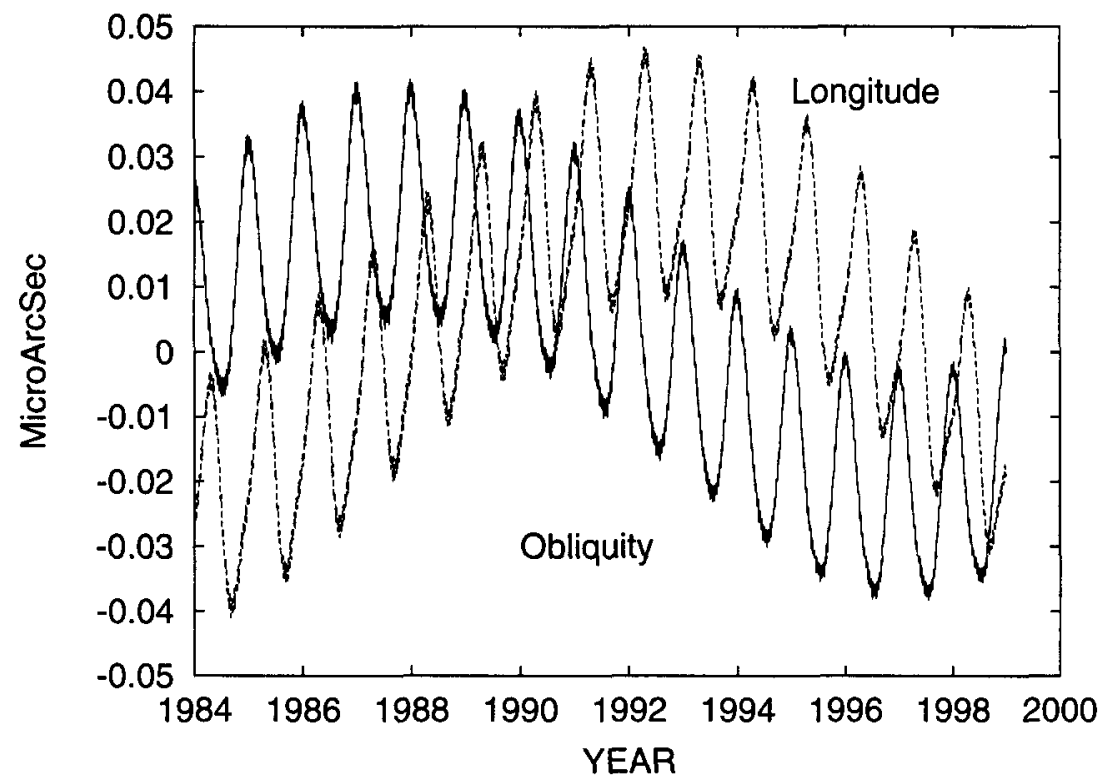

Figure 1. Error of Numerical Convolution.

Note: Shown are the components in $\Delta \epsilon$ and $\Delta \psi \sin \epsilon_{0}$ for the difference between the numerically and analytically convolved nutations of nonrigid Earth. Here the rigid Earth theory is that of Kinoshita (1977) while the transfer function is that of Wahr (1981).

\section{Example of Numerical Convolution}

In order to evaluate our method, we did a series of comparisons with the analytical method of convolution expressed in Eq. (5). Let us show an example.

First, we fixed the rigid Earth nutation $z$ as that of Kinoshita (1977). We denote the nutation convolved analytically by $\zeta_{A}$ and that done numerically by the new method by $\zeta_{N}$. Here, in order to make the analytical convolution rigorous (See discussion in Section 2.1 and Table 2), we dropped the mixed secular terms from the table of Kinoshita and removed the higher-order trends from the Delauney arguments. Then we set the stepsize of the tabulation as 1.5 hours, namely 16 points per day. As for the integro-diffrential operations, we adopted the 9-point central difference formula, Eq. (13), to evaluate the numerical differentiation and the 8-point symmetric integration formula, Eq. (26), to perform the numerical integration.

Figure 1 illustrates the resulting residuals $\zeta_{N}-\zeta_{A}$ for the case when the period of comparison was $15 \mathrm{yr}$, actually $1984-1999$, the period when the observed nutations were determined from the VLBI observations. Figure 2 is its close-up for the first few years. From the term-by-term comparison, we learned that the observed errors mostly came from the truncation error of the adopted integration formula for the Free Core Nutation mode. See also Table 2. We remark that this error can be reduced further by choosing a smaller stepsize or by selecting a higher order formula. Anyway, as is clearly shown in the figures, 


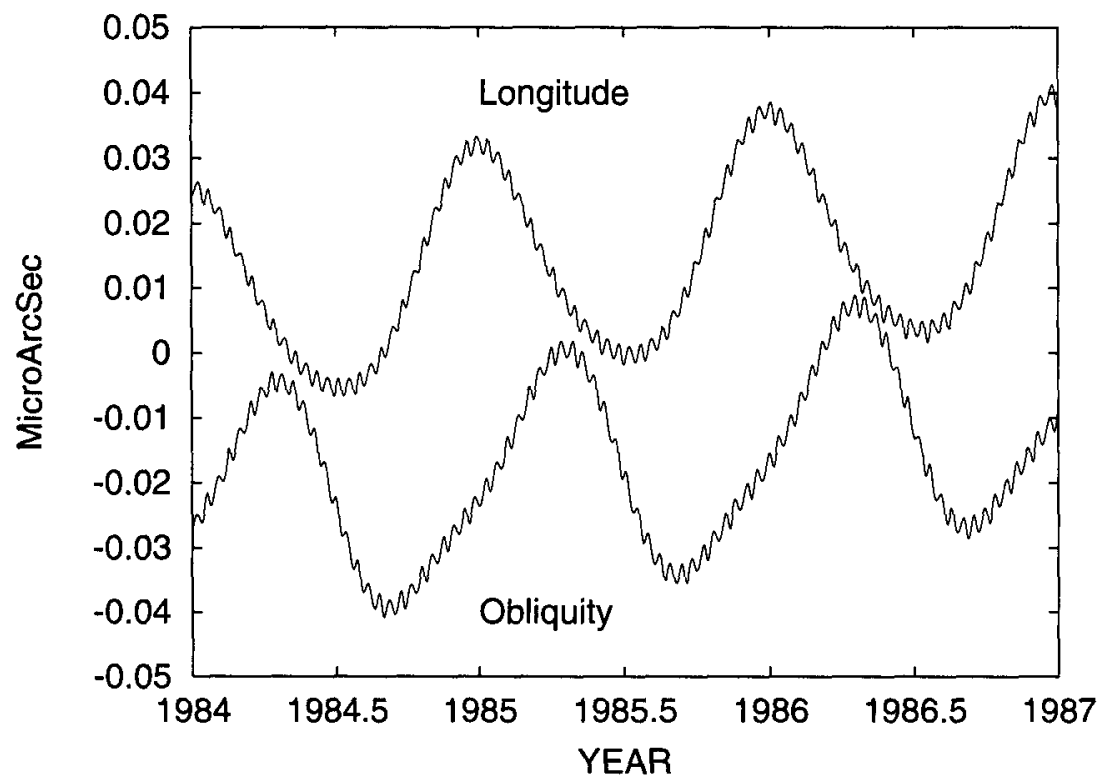

Figure 2. Error of Numerical Convolution: Close-Up. Note: Same as Figure 1 but shown for the first few years.

the error of numerical convolution is of the order of 10 nano arcseconds, which we think sufficiently small.

\section{Conclusion}

We developed a numerical method of convolution. The method deploys the integro-differential operations in the time domain numerically instead of multiplying a rational function in the frequency domain. Namely the multiplication of a polynomial in the frequency domain is translated into the numerical differentiations in the time domain and that of fractions into the numerical integrations with a suitable kernel in the time domain. In executing the numerical integrations, the unknown integral constants, which corresponds to the free oscillation component, are determined by a least-squares method to fit the observational data. The numerical differentiation and integration are effectively done by means of the symmetric difference and integral formulas. Numerical tests showed that the method is sufficiently precise to reproduce the analytically convolved nutation at the level of 10 nano arcseconds. Since the method only requires the rigid Earth nutation theory to be expressed as a numerical table of time, it enables us to create a purely numerical theory of nutation of the nonrigid Earth.

\section{References}

Bretagnon, P., Rocher, P., and Simon J.-L., 1997, Theory of the Rotation of the 
Rigid Earth, Astron. Astrophys., 319, 305-317.

Dehant, V., Arias, F., Bizouard, Ch., Bretagnon, P., Brzeziński, A., Buffett, B., Capitaine, N., Defraigne, P., DeViron, O., Feissel, M., Fliegel, H., Forte, A., Gambis, D., Getino, J., Gross, R., Herring, T., Kinoshita, H., Klioner, S., Mathews, P.M., McCarthy, D., Moisson, X., Petrov, S., Ponte, R.M., Roosbeek, F., Salstein, D., Schuh, H., Seidelmann, K., Soffel, M., Souchay, J., Vondrák, J., Wahr, J.M., Wallace, P., Weber, R., Williams, J., Yatskiv, Y., Zharov, V., 1999, Considerations Concerning the Non-Rigid Earth Nutation Theory, Celest. Mech. Dynamical Astron., $\mathbf{7 2}, 245-310$.

Dehant, V. and Defraigne, P., 1997, New Transfer Functions for Nutations of a Non-Rigid Earth, J. Geophys. Res., 104, B3, 4861-4875.

Defraigne, P., Dehant, V., and Paquet, P., 1995, Link between the RetrogradePrograde Nutations and Nutations in Obliquity and Longitude, Celest. Mech. Dynamical Astron., 62, 363-376.

Getino, J., Martin, P., and Farto, J.M., 1999, Improved Nutation Series for the Non-Rigid Earth with a Precise Adjustment of Parameters with Nonlinear Dependence, Celest. Mech. Dynamical Astron., 74, 153-162.

Herring, T.A., 1995, A Priori Model for the Reduction of the Nutation Observations, Highlights of Astronomy, 10, 222-227.

Kinoshita, H., 1977, Theory of the Rotation of the Rigid Earth, Celestial Mechanics, 15, 277-326.

Kinoshita, H. and Souchay, J., 1990, The Theory of the Nutation for the Rigid Earth Model at the Second Order, Celestial Mechanics, 48, 187-265.

Mathews, P.M., Buffett, B.A., Herring, T.A., and Shapiro I.I., 1991a, Forced Nutations of the Earth: Influence of Inner Core Dynamics. I. Theory, $J$. Geophys. Res., 96 B5, 8219-8242.

Mathews, P.M., Buffett, B.A., Herring, T.A., and Shapiro I.I., 1991b, Forced Nutations of the Earth: Influence of Inner Core Dynamics. II. Numerical Results and Comparisons, J. Geophys. Res., 96 B5, 8243-8258.

Mathews, P.M., Buffett, B.A., and Herring, T.A., 1999, The Magnetic Coupling Contribution to Nutation, in Proc. Journées Systèmes de Référence 1998, Paris, France.

Moritz, H. and Mueller, I.I., 1987, Earth Rotation, Ungar, New York.

Roosbeek, F. and Dehant, V., 1998, RDAN97: an Analytical Development of Rigid Earth Nutation Series Using the Torque Approach, Celest. Mech. Dynamical Astron., 70, 215-253.

Schastok, J., 1997, A New Nutation Series for a More Realistic Model Earth, Geophys. J. Int., 130, 137-150. 
Seidelmann, P.K., 1982, 1980 IAU Theory of Nutation: the Final Report of the IAU Working Group on Nutation, Celestial Mechanics, 27, 79-106.

Wahr, J.M., 1981, The Forced Nutations of an Elliptical, Rotating, Elastic and Oceanless Earth, Geophys. J. R. Astron. Soc., 64, 705-727. 